Report

\title{
Association of genetic variation in tamoxifen-metabolizing enzymes with overall survival and recurrence of disease in breast cancer patients
}

\author{
Susan A. Nowell ${ }^{1}$, Jiyoung Ahn ${ }^{1}$, James M. Rae ${ }^{2}$, Joshua O. Scheys ${ }^{2}$, Andrew Trovato ${ }^{3}$, \\ Carol Sweeney ${ }^{4}$, Stewart L. MacLeod ${ }^{5}$, Fred F. Kadlubar ${ }^{6}$, and Christine B. Ambrosone ${ }^{1}$ \\ ${ }^{1}$ Department of Epidemiology, Roswell Park Cancer Institute, Buffalo, NY; ${ }^{2}$ Department of Internal Medicine, \\ University of Michigan School of Medicine; ${ }^{3}$ Merck and Company, Rahway, NJ; ${ }^{4}$ Department of Family and \\ Preventive Medicine, University of Utah, Salt Lake City, UT; ${ }^{5}$ University of Arkansas for Medical Sciences; ${ }^{6}$ Division \\ of Molecular Epidemiology, National Center for Toxicological Research, Jefferson, AR, USA
}

Key words: breast cancer, CYP2D6, genetic variation, SULT1A1, tamoxifen, UGT2B15

\begin{abstract}
Summary
Tamoxifen has been a mainstay of adjuvant therapy for breast cancer for many years. We sought to determine if genetic variability in the tamoxifen metabolic pathway influenced overall survival in breast cancer patients treated with tamoxifen. We examined functional polymorphisms in CYP2D6, the P450 catalyzing the formation of active tamoxifen metabolites, and UGT2B15, a Phase II enzyme facilitating the elimination of active metabolite in a retrospective study of breast cancer patients. We also examined whether the combination of variant alleles in SULT1A1 and UGT2B15 had more of an impact on overall survival in tamoxifen-treated patients than when the genes were examined separately.

We conducted a retrospective study using archived paraffin blocks for DNA extraction and data from pathology reports and hospital tumor registry data for information on clinical characteristics, treatment, and outcomes (162 patients receiving tamoxifen and 175 who did not). Genotypes for CYP2D6 and UGT2B15 were obtained and Cox proportional hazards modeling was performed.

After adjusting for age, race, stage of disease at diagnosis, and hormone receptor status, we found no significant association between CYP2D6 genotype and overall survival in either group of breast cancer patients. Tamoxifentreated patients with UGT2B15 high activity genotypes had increased risk of recurrence and poorer survival. When UGT2B15 and SULT1A1 'at-risk' alleles were combined, women with two variant alleles had significantly greater risk of recurrence and poorer survival than those with common alleles. These studies indicate that genetic variation in Phase II conjugating enzymes can influence the efficacy of tamoxifen therapy for breast cancer.
\end{abstract}

\section{Introduction}

For over two decades, tamoxifen has been the gold standard of endocrine therapy for estrogen receptorpositive breast cancer [1], and its use has contributed to the decline in breast cancer mortality. Analysis performed by the Early Breast Cancer Trialist's Collaborative Group in 1998 demonstrated that breast cancer patients treated with 5 years of tamoxifen had a $47 \%$ reduction in recurrence of disease after 10 years of follow-up and treatment was also associated with a $47 \%$ reduction in the occurrence of contralateral breast cancer [2]. Tamoxifen has mixed estrogenic and antiestrogenic properties; it is antiestrogenic in breast tumors [1], while the estrogenic properties are responsible for preservation of bone mineral density in postmenopausal women $[3,4]$, decreasing low-density lipoprotein cholesterol $[5,6]$ and lowering the risk of myocardial infarction [7].
Competition by tamoxifen and its metabolites with estradiol for occupancy of the estrogen receptor, thus blocking estradiol-mediated cellular proliferation, is considered to be one of the major mechanisms of tamoxifen's pharmacological action. The metabolism of tamoxifen is complex, with tamoxifen undergoing both oxidative and conjugative reactions. In the liver, tamoxifen is converted to its major metabolite, N-desmethyltamoxifen, by cytochrome P450 3A4 (CYP3A4), but this metabolite has low affinity for the estrogen receptor. Two minor metabolites of tamoxifen, 4-hydroxytamoxifen (4-OH-TAM) and 4-hydroxy-Ndesmethyltamoxifen (endoxifen) are produced in a reaction primarily mediated by cytochrome CYP2D6 [8], although other P450s are capable of forming 4-OH TAM. These metabolites have much greater affinity for the estrogen receptor than either tamoxifen or $\mathrm{N}$-desmethyltamoxifen, and are considered to be the active metabolites of tamoxifen $[9,10]$. CYP2D6 is 
highly polymorphic in humans, with over 70 allelic variants described. However, the CYP2D6 'poor metabolizer' phenotype can be attributed to the presence of five alleles [11], with the CYP2D6*4 allele being common in Caucasians. It is possible that, given the role of CYP2D6 in the production of 4-OH TAM and endoxifen, genetic variation in this enzyme could influence treatment outcomes.

4-OH TAM is a substrate for conjugation reactions catalyzed by sulfotransferase isoform 1A1 (SULT1A1) and UDP-glucuronosyltransferase isoform 2B15 (UGT2B15) [12]. Both of these enzymes have functional genetic polymorphisms; the SULT1A1*2 variant allele is associated with decreased catalytic activity $[13,14]$ and the UGT2B15 $\mathrm{Asp}^{85} \operatorname{Tyr}\left(\mathrm{UGT} 2 \mathrm{~B} 15^{*} 1 /{ }^{* 2}\right)$ variant is located within the putative substrate recognition site of the enzyme and is associated with increased velocity of reaction [15]. We have shown previously that the low activity SULT1A1*2 variant was associated with significantly poorer overall survival in breast cancer patients receiving tamoxifen [16]. Patients who were homozygous for the variant SULT1A $1 * 2$ allele had an almost threefold increase in hazard of death compared to patients who were heterozygous or homozygous for the SULT1A1*1 common allele. To test the specificity of the association, the SULT1A1*2 genotype was also examined in a group of patients whose therapies did not include tamoxifen; there was no association between SULT1A1 genotype and survival in this group. In light of these findings, we sought to determine if polymorphisms in other tamoxifen-metabolizing enzymes, particularly CYP2D6, which produces the active metabolites, and UGT2B15, which is involved in the elimination of active metabolites, influenced overall survival in the same study population. Additionally, we examined the combined effects of UGT2B15 and SULT1A1 genetic variation on therapeutic outcomes.

\section{Patients and methods}

\section{Study Population}

The study population has been previously described in detail in [16]. Patients who received their first course of adjuvant treatment for primary invasive breast cancer at the Arkansas Cancer Research Center, University of Arkansas for Medical Sciences (UAMS) between the years 1985 and 1996 were identified from hospital tumor registry records; patients with a prior history of cancer were excluded from the study. Tumor registry records and pathology reports were used to obtain information concerning age, stage at diagnosis, hormone receptor status, race, date and type of therapy received (chemotherapy, radiotherapy, surgery, hormonal treatment) and followup data. The final study population was comprised of a total of 162 patients who received tamoxifen and 175 who did not receive hormonal therapy. The study protocol was approved by the Institutional Review Board of UAMS.

\section{DNA extraction and genotyping}

Paraffin-embedded archived tissues were used as a source of DNA for genotyping. A block containing normal lymph nodes was selected if available; if normal lymph nodes were not available, a block containing other tissue noted as histologically normal was used. Sections $(50-\mu \mathrm{m})$ were cut from a paraffin block containing normal tissue, the tissue was deparaffinized and DNA was extracted using a commercially available kit (Qiagen, Valencia, CA), as described previously [17].

\section{Cytochrome P $4502 D 6$ (CYP2D6) taqman allelic discrimination assay}

Patients were genotyped for CYP2D6*3, CYP2D6*4 and CYP2D6*6 polymorphisms using the Applied Biosystems' Taqman ${ }^{\circledR}$ Allelic Discrimination Assay according to the manufacturer's instructions. Briefly, $10 \mathrm{ng}$ DNA was added to a $5 \mu \mathrm{l}$ reaction containing forward and reverse primers along with two allele specific labeled probes (one wild-type and one variant allele specific). The PCR and fluorescence measurements were performed using the ABI Prism ${ }^{\circledR} 7900$ HT sequence detection system.

\section{UGT2B15 genotype determination}

An overlapping, bidirectional PCR assay to simultaneously amplify specific UGT2B15 alleles was developed based on the Bi-PASA method of Liu et al. [18]. The region flanking the polymorphic base pair was amplified in a PCR reaction using outer primers as reported by Levesque et al. [15] (UGT2B15 forward primer: 5'-G ACTGTGTTGACATCTTCGGCTTCT-3'; UGT2B15 reverse primer: 5'-CCAGTAGCTCACCACAGGGAT TAAG- $3^{\prime}$ ). In addition to the outer primers, the PCR reaction incorporates allele specific inner primers that amplify products in different directions, producing different size products depending on the identity of the polymorphic base. The inner allele specific primers were forward: 5'-GGGGCGGGGCCTACATCTTTAACT AAAAATG-3' and reverse: 5'-CGGGGCGGGGCA GAAGAGAATCTTCCAAATA- ${ }^{\prime}$. These primers incorporate $\mathrm{GC}$ rich tails that allow a switch from low efficiency priming with genomic DNA as template, to high efficiency amplification using previously amplified PCR product as template. The amplification was performed in a $50 \mu \mathrm{l}$ volume containing $100 \mathrm{ng}$ of genomic DNA. $200 \mu \mathrm{M}$ of each dNTP, $1 \times$ PCR buffer (Promega), $2.5 \mathrm{mM} \mathrm{MgCl}_{2}, 0.25 \mu \mathrm{M}$ outer primers, $0.5 \mu \mathrm{M}$ inner forward primer, $1.0 \mu \mathrm{M}$ reverse inner primer, 2.5 units Taq polymerase and $500 \mathrm{ng}$ Taq-Start antibody. After initial denaturation at $95^{\circ} \mathrm{C}$ for $4 \mathrm{~min}$, the samples were subjected to 35 cycles of $95^{\circ} \mathrm{C}$ for $30 \mathrm{~s}$, $60{ }^{\circ} \mathrm{C}$ for $30 \mathrm{~s}$ and $72{ }^{\circ} \mathrm{C}$ for $30 \mathrm{~s}$, followed by a final extension step of $7 \mathrm{~min}$ at $72^{\circ} \mathrm{C}$. Products of the PCR reaction were detected by electrophoresis on a $4 \%$ 
Metaphor agarose gel and visualized by ethidium bromide staining and UV transillumination.

\section{Statistical analysis}

We first evaluated relationships between patient and tumor characteristics by genotype using $\chi^{2}$ test. Survival time was calculated as the time from diagnosis to death or to the last contact date for living subjects. For analysis of disease-free survival, time from disease-free date to recurrence, death, or last follow-up was calculated. Person-years were calculated as the sum of survival times for all subjects within a group. Crude associations between genotypes for CYP2D6, UGT2B15, and SULT1A1 and overall survival and recurrence-free survival were evaluated using Kaplan-Meier survival function with log-rank tests. Both heterozygote and homozygote variant genotypes were assessed separately in relation to the referent common genotype. Relative risks were estimated by calculating hazard ratios (HR) from Cox proportional hazard models. The final model was adjusted for age, stage with node status at diagnosis, race, ER status, and PR status. To examine the joint effects of genotypes, we created dummy variables, combining genotypes for UGT2B15 and SULT1A1, using data on SULT1A1 that was previously reported [16]. Analyses were conducted using SAS version 8.2.

\section{Results}

We obtained normal tissue samples and complete baseline information for 165 eligible study subjects who had received adjuvant tamoxifen therapy, and for 172 who had not been treated with tamoxifen. The characteristics of the study population are presented in Table 1 and have been described in detail elsewhere [16]. When characteristics were considered according to CYP2D6, UGT2B15 or SULT1A1 genotype, there was no influence of genotype on patient characteristics. Genotype frequencies did not differ by race. Genotype frequencies for CYP2D6*4 were 0.15 for both races. Genotype frequencies for UGT2B15*2 were 0.47 for Caucasians and 0.49 for African-Americans and genotype frequencies for SULT1A $1 * 2$ were 0.35 for Caucasians and 0.33 for African-Americans.

Since other CYP2D6 alleles (*3 and *6) were infrequent in our study population, we performed our analyses for one or more CYP2D6*4 variant alleles compared to common alleles. After adjusting for age, stage of disease, ethnicity and hormone receptor status, Cox proportional hazards modeling showed no association between CYP2D6*4 genotype and overall survival of breast cancer patients receiving tamoxifen (Hazard ratio $(\mathrm{HR})=0.77,95 \%$ CI $0.32-1.81$, Table 2). When the same analysis was performed for the group of patients whose therapy did not include tamoxifen, there was also no association between CYP2D6 genotype and overall survival $(\mathrm{HR}=0.79$,
Table 1. Demographic information and pathological characteristics of study participants and distributions of characteristics

\begin{tabular}{|c|c|c|}
\hline & $n(n=337)$ & $\%$ \\
\hline \multicolumn{3}{|l|}{ Age at Diagnosis } \\
\hline$<50$ year & 139 & $(41)$ \\
\hline$>50$ year & 198 & $(59)$ \\
\hline \multicolumn{3}{|l|}{ Race } \\
\hline Caucasian & 272 & $(81)$ \\
\hline African-American & 65 & (19) \\
\hline \multicolumn{3}{|l|}{ Stage with node status } \\
\hline 1 & 106 & (31) \\
\hline 2 negative & 71 & $(21)$ \\
\hline 2 positive & 95 & $(28)$ \\
\hline 3 & 49 & (15) \\
\hline 4 & 16 & (5) \\
\hline \multicolumn{3}{|l|}{ ER status } \\
\hline Positive & 226 & (67) \\
\hline Negative & 111 & (33) \\
\hline \multicolumn{3}{|l|}{ PR status } \\
\hline Positive & 174 & $(52)$ \\
\hline Negative & 163 & $(48)$ \\
\hline \multicolumn{3}{|l|}{ Treatment } \\
\hline Chemo only & 98 & (29) \\
\hline Radiation only & 21 & (6) \\
\hline Chemo/radiation & 53 & (16) \\
\hline Tamoxifen only & 48 & (14) \\
\hline Radiation/tamoxifen & 37 & (11) \\
\hline Chemo/tamoxifen & 48 & (14) \\
\hline Chemo/radiation/tamoxifen & 32 & (10) \\
\hline \multicolumn{3}{|l|}{ Vital status } \\
\hline Alive & 237 & (70) \\
\hline Death & 100 & (30) \\
\hline Cancer cause death & 61 & (61) \\
\hline Death with other reasons & 7 & (7) \\
\hline Unknown & 32 & (32) \\
\hline \multicolumn{3}{|l|}{ Recurrence } \\
\hline None & 243 & $(72)$ \\
\hline Recurrence or death & 84 & $(25)$ \\
\hline Never disease free & 10 & (3) \\
\hline Median survival year & 4.58 year & \\
\hline
\end{tabular}

95\% CI 0.42-1.26). We also considered the association between progression-free survival and CYP2D6 genotype in tamoxifen-treated patients. After excluding 11 patients who were never disease-free, there was no association between recurrence of disease and CYP2D6 genotype in tamoxifen-treated patients $(\mathrm{HR}=0.67,95 \%$ CI $0.33-1.35)$ or patients whose therapy did not include tamoxifen $(\mathrm{HR}=0.69,95 \% \mathrm{CI}$ 0.40-1.18, Table 2).

When UGT2B15 genotype was considered, there was no significant association between genotype and survival in either the patient group receiving tamoxifen or the group whose therapy did not include tamoxifen (Table 3). When recurrence of disease was examined according to UGT2B15 genotype, there was a 
Table 2. Overall and progression-free survival of breast cancer patients according to CYP2D6 Genotype

\begin{tabular}{|c|c|c|c|c|c|}
\hline Genotype & Cases & Deaths & Person-years & Deaths/person-years & $\operatorname{HR}^{\mathrm{a}}(95 \% \mathrm{CI})$ \\
\hline \multicolumn{6}{|l|}{ Overall survival } \\
\hline Tamoxifen & 162 & 34 & & & \\
\hline Cyp2d6 wt/wt & 114 & 27 & 593 & 0.046 & 1 (Ref) \\
\hline Cур $2 \mathrm{~d} 6 * 4 / * 4+* 4 / \mathrm{wt}$ & 48 & 7 & 253 & 0.028 & $\begin{array}{l}0.77(0.32-1.81) \\
p \text { trend }=0.51\end{array}$ \\
\hline No tamoxifen & 175 & 66 & & & \\
\hline Cyp2d6 wt/wt & 126 & 48 & 596 & 0.080 & 1 (Ref) \\
\hline Cyp2d $6 * 4 / * 4+* 4 /$ wt & 49 & 18 & 286 & 0.063 & $\begin{array}{l}0.79(0.42-1.26) \\
p \text { trend }=0.26\end{array}$ \\
\hline \multicolumn{6}{|l|}{ Progression-free survival ${ }^{\mathrm{b}}$} \\
\hline Tamoxifen & 160 & 48 & & & \\
\hline Cyp2d6 wt/wt & 112 & 38 & 524 & 0.073 & 1 \\
\hline Cyp 2 d $6 * 4 / * 4+* 4 /$ wt & 48 & 10 & 524 & 0.019 & $\begin{array}{l}0.67(0.33-1.35) \\
p \text { trend }=0.19\end{array}$ \\
\hline No tamoxifen & 166 & 71 & & & \\
\hline Сур2d6 wt/wt & 120 & 53 & 480 & 0.110 & 1 \\
\hline Cyp2d $6 * 4 / * 4+* 4 /$ wt & 46 & 18 & 240 & 0.075 & $\begin{array}{l}0.69(0.40-1.18) \\
p \text { trend }=0.19\end{array}$ \\
\hline
\end{tabular}

${ }^{\mathrm{a}} \mathrm{HRs}$ for fully adjusted model: age, stage with node status at diagnosis, race, ER status, and PR status.

${ }^{\mathrm{b}}$ Eleven subjects were excluded because they were never disease-free.

Table 3. Overall and progression-free survival of breast cancer patients according to UGT2B15 Genotype

\begin{tabular}{|c|c|c|c|c|c|c|}
\hline Genotype & Cases & Deaths & Person-years & $\begin{array}{l}\text { Deaths } \\
\text { /person-years }\end{array}$ & $\operatorname{HR}^{\mathrm{a}}(95 \% \mathrm{CI})$ & \\
\hline \multicolumn{7}{|l|}{ Overall survival } \\
\hline Tamoxifen & 165 & 34 & & & & \\
\hline UGT2B $15 * 1 / * 1$ & 32 & 5 & 159 & 0.032 & 1 & \\
\hline $\mathrm{UGT} 2 \mathrm{~B} 15 * 1 / * 2$ & 108 & 24 & 575 & 0.042 & 1.19 & $(0.44-3.22)$ \\
\hline UGT2B $15 * 2 / * 2$ & 25 & 5 & 127 & 0.040 & $\begin{array}{l}1.48 \\
p \text { trend }=0.55\end{array}$ & $(0.42-5.27)$ \\
\hline No Tamoxifen & 172 & 66 & & & & \\
\hline UGT2B $15 * 1 / * 1$ & 36 & 14 & 187 & 0.075 & 1 & \\
\hline UGT2B $15 * 1 / * 2$ & 110 & 43 & 552 & 0.078 & 1.15 & $0.62-2.12$ \\
\hline UGT2B $15 * 2 / * 2$ & 26 & 9 & 126 & 0.071 & $\begin{array}{l}1.11 \\
p \text { trend }=0.78\end{array}$ & $0.47-2.58$ \\
\hline \multicolumn{7}{|c|}{ Progression-free survival $^{\mathrm{b}}$} \\
\hline Tamoxifen & 163 & 49 & & & & \\
\hline UGT2B $15 * 1 / * 1$ & 32 & 7 & 147 & 0.041 & & \\
\hline UGT2B $15 * 1 / * 2$ & 106 & 33 & 511 & 0.039 & $0.61-3.12$ & \\
\hline UGT2B $15 * 2 / * 2$ & 25 & 9 & 112 & 0.045 & $\begin{array}{l}0.70-5.11 \\
p \text { trend }=0.11\end{array}$ & \\
\hline No Tamoxifen & 164 & 70 & & & & \\
\hline UGT2B $15 * 1 / * 1$ & 33 & 12 & 161 & 0.05 & & \\
\hline $\mathrm{UGT} 2 \mathrm{~B} 15 * 1 / * 2$ & 106 & 48 & 448 & 0.107 & $0.70-2.49$ & \\
\hline UGT2B $15 * 2 / 2$ & 25 & 10 & 103 & 0.097 & $\begin{array}{l}0.46-2.60 \\
p \text { trend }=0.63\end{array}$ & \\
\hline
\end{tabular}

${ }^{\text {a }}$ HRs for fully adjusted model: age, stage with node status at diagnosis, race, ER status, and PR status.

${ }^{\mathrm{b}}$ Ten subjects were excluded because they were never disease-free; genotype data was missing for one subject.

non-significant trend toward increased recurrence of disease with increasing numbers of UGT2B $15 * 2$ alleles $(\mathrm{HR}=1.56,95 \%$ CI $0.68-3.61$ for UGT2B $15 * 1 / 2$ and $\mathrm{HR}=2.27$, 95\% CI $0.82-6.28$ for UGT2B $15 * 2 * 2$, ptrend $=0.11)$. In patients who did not receive tamoxifen, there was no evidence of a trend associated with increasing numbers of UGT2B $15 * 2$ alleles and recurrence of disease (Table 3). 
Since SULT1A1 and UGT2B15 have overlapping substrate specificity for 4-OH TAM, and both are Phase II detoxification enzymes, we examined combinations of variant alleles to determine if there was an additive effect with the genotypes and outcomes from tamoxifen therapy. Tests for statistical interaction between genotypes showed no interaction between SULT1A1 and UGT2B15 in either the group receiving tamoxifen $(p=0.56)$ or those not receiving tamoxifen $(p=0.58)$. Earlier studies from our laboratory have shown that enzymatic activity does not differ between SULT1A $1 * 1 / * 1$ and SULT1A1*1/*2, while enzymatic activity associated with SULT1A $1 * 2 / * 2$ is significantly lower than that associated with the other two genotypes [14]. Therefore, the referent category included SULT1A $1 * 1 / * 1$ and SULT1A $1 * 1 / 2$ in combination with UGT2B $15 * 1 / * 1$ genotype. Genotypes were assigned to the two variant category if they contained SULT1A $1 * 2 / * 2$ in combination with UGT2B $15 * 1 / * 2$ or UGT2B $15 * 2 / * 2$. All other possible allele combinations were categorized as possessing one variant allele. When overall survival was considered in the tamoxifen-treated patients, there was a significant trend toward decreased overall survival with increasing numbers of variant alleles $(\mathrm{HR}=1.13,95 \% \mathrm{CI} 0.37-3.44$ for one variant and $\mathrm{HR}=4.40,95 \%$ CI $1.17-16.55$ for two variants, ptrend $=0.03$, Table 4 ), although sample size was small, resulting in unstable risk estimates. There was no effect of numbers of variant alleles in patients whose therapy did not include tamoxifen $(\mathrm{HR}=1.08,95 \%$ CI $0.56-2.07$ for one variant and $\mathrm{HR}=0.84,95 \% \mathrm{CI} 0.29-2.43$ for two variant alleles.

The Kaplan-Meier function for survival by combined UGT2B15 and SULT1A1 genotypes is shown in Figure 1A for tamoxifen-treated patients and Figure 1B for patients not receiving tamoxifen. The proportion surviving at 5 years among tamoxifen-treated patients was $0.88(95 \%$ CI $0.81-2.47)$ for those with one variant allele and $0.60(95 \%$ CI $0.33-1.24)$ in the group with two variant alleles $(p=0.003)$. There was no difference in survival according to variant genotype among patients who were not treated with tamoxifen; the proportion of patients not treated with tamoxifen who were alive at 5 years was $0.61(95 \%$ CI $0.52-$ 1.62) for one variant allele, and $0.78(95 \%$ CI $0.57-$ $1.90)$ for two variants $(p=0.81)$. When the group of

Table 4. Overall survival of breast cancer patients according to UGT2B15 and SULT1A1 Genotypes

\begin{tabular}{|c|c|c|c|c|c|}
\hline Genotype & Cases & Deaths & Person-years & Death/person-years & $\mathrm{HR}^{\mathrm{a}}(95 \% \mathrm{CI})$ \\
\hline \multicolumn{6}{|l|}{ Overall survival } \\
\hline Tamoxifen & 160 & 34 & & & \\
\hline Both referent ${ }^{\mathrm{b}}$ & 23 & 4 & 122 & 0.033 & 1 \\
\hline 1 variant allele ${ }^{c}$ & 121 & 23 & 652 & 0.035 & $1.13(0.37-3.44)$ \\
\hline \multirow[t]{2}{*}{2 variant alleles ${ }^{\mathrm{d}}$} & 16 & 7 & 67 & 0.104 & $4.40(1.17-16.55)$ \\
\hline & & & & & $p$ trend $=0.03$ \\
\hline No tamoxifen & 171 & 65 & & & \\
\hline Both referent & 31 & 12 & 159 & 0.076 & 1 \\
\hline 1 variant allele & 124 & 48 & 575 & 0.084 & $1.08(0.56-2.07)$ \\
\hline \multirow[t]{2}{*}{2 variant alleles } & 16 & 5 & 127 & 0.040 & $0.84(0.29-2.43)$ \\
\hline & & & & & $p$ trend $=0.87$ \\
\hline \multicolumn{6}{|c|}{ Progression-free survival ${ }^{\mathrm{e}}$} \\
\hline Tamoxifen & 158 & 49 & & & \\
\hline Both referent & 23 & 6 & 111 & 0.054 & 1 \\
\hline 1 variant allele & 120 & 36 & 585 & 0.062 & $1.54(0.62-3.82)$ \\
\hline \multirow[t]{2}{*}{2 variant alleles } & 15 & 7 & 56 & 0.125 & $3.79(1.18-12.15)$ \\
\hline & & & & & $p$ trend $=0.03$ \\
\hline No tamoxifen & 163 & 69 & & & \\
\hline Both referent & 28 & 10 & 147 & 0.068 & 1 \\
\hline 1 variant allele & 119 & 52 & 511 & 0.102 & $1.25(0.63-2.51)$ \\
\hline \multirow[t]{2}{*}{2 variant alleles } & 16 & 7 & 112 & 0.063 & $1.31(0.50-3.45)$ \\
\hline & & & & & $p$ trend $=0.35$ \\
\hline
\end{tabular}

${ }^{\mathrm{a}} \mathrm{HRs}$ for fully adjusted model: age, stage with node status at diagnosis, race, ER status, and PR status.

${ }^{\mathrm{b}}$ Both referent: SULT1A $1 * 1 /{ }^{*} 1: \mathrm{UGT} 2 \mathrm{~B} 15 * 1 /{ }^{*} 1$ or SULT1A $1 * 1 / * 2: \mathrm{UGT} 2 \mathrm{~B} 15^{*} 1 /{ }^{*} 1$.

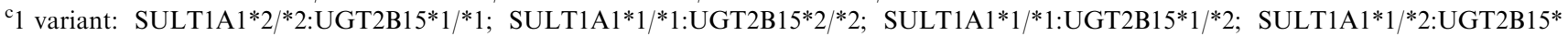
$1 / * 2 ; \operatorname{SULT} 1 \mathrm{~A} 1 * 1 / * 2: \mathrm{UGT} 2 \mathrm{~B} 15 * 2 / * 2$.

dTwo variants: SULT1A $1 * 2 / * 2: \mathrm{UGT} 2 \mathrm{~B} 15 * 1 / * 2 ; \mathrm{SULT} 1 \mathrm{~A} 1 * 2 / * 2: \mathrm{UGT} 2 \mathrm{~B} 15 * 2 / * 2$.

${ }^{\mathrm{e}}$ Ten subjects were excluded because they were never disease-free; genotype data was missing for one subject.

*SULT1A1 genotype data was missing for two tamoxifen-treated subjects and four subjects who did not receive tamoxifen. 


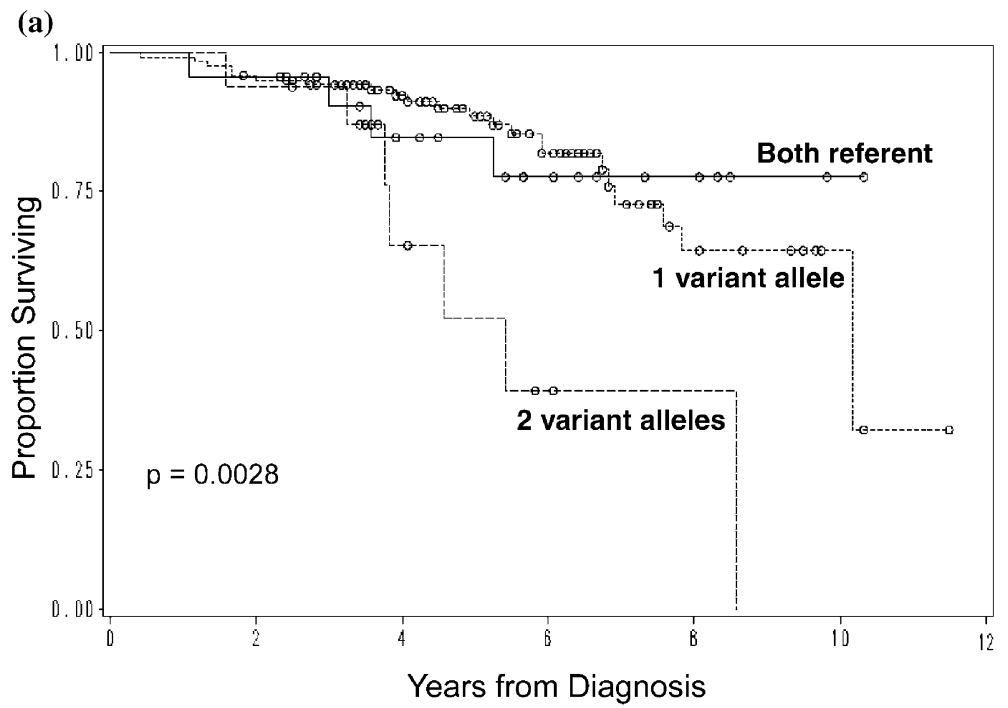

(b)

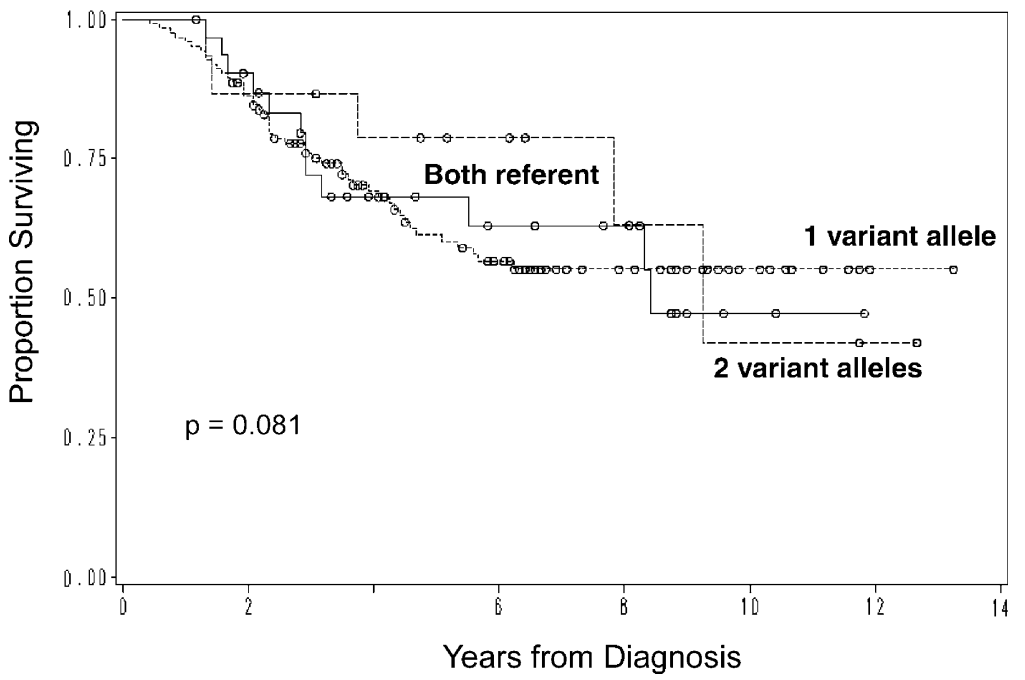

Figure 1. (a) O gdverall survival of breast cancer by combined UGT2B15 and SULT1A1 genotypes [35]. Patients receiving tamoxifen therapy; (b) patients not receiving tamoxifen as part of their treatment for breast cancer.

women who did not receive tamoxifen was restricted to patients with ER positive tumors $(n=82)$, there was also no survival difference according to UGT2B15 and SULT1A1 genotype in this subgroup $(p=0.44)$. When progression free survival was considered, Kaplan-Meier survival function showed a significant effect on numbers of variant alleles on recurrence of disease (Figure 2a). The proportion of patients free of progression at 5 years was 0.78 (95\% CI $0.69-2.14)$ for those with one variant allele and $0.50(95 \%$ CI $0.22-$ $0.93)$ for two variants $(p=0.05)$. In the patient group not receiving tamoxifen (Figure $2 b$ ), the proportion of patients free of progression at 5 years was $0.53(95 \%$ CI $0.44-1.38)$ with one variant allele and $0.57(95 \%$ CI 0.31-1.18) for patients with two variant alleles $(p=0.74)$. The addition of UGT2B15 genotype to the model produced a significant change of log likelihood ratio, compared to a model with SULT1A1 genotype alone $(p=0.01)$.

\section{Discussion}

A previous study from our laboratory demonstrated that a common genetic polymorphism in SULT1A1, a Phase II enzyme catalyzing the sulfation of 4-OH TAM, was associated with poorer overall survival in breast cancer patients receiving tamoxifen therapy [16]. In the current study, and using the same study population as the previous study, we demonstrated that genetic variation in the Phase II enzymes, SULT1A1 and UGT2B15, alone or in combination was associated with overall survival and recurrence of disease. In contrast, genetic variation in CYP2D6, which catalyzes the Phase I metabolism of tamoxifen to produce endoxifen and 4-OH TAM, had no apparent effect on overall survival or recurrence of disease in tamoxifen-treated patients.

The majority of breast cancers occur in postmenopausal women [19] whose tumors express the estrogen and/or progesterone receptors $[1,20,21]$ and tamoxifen 
(a)

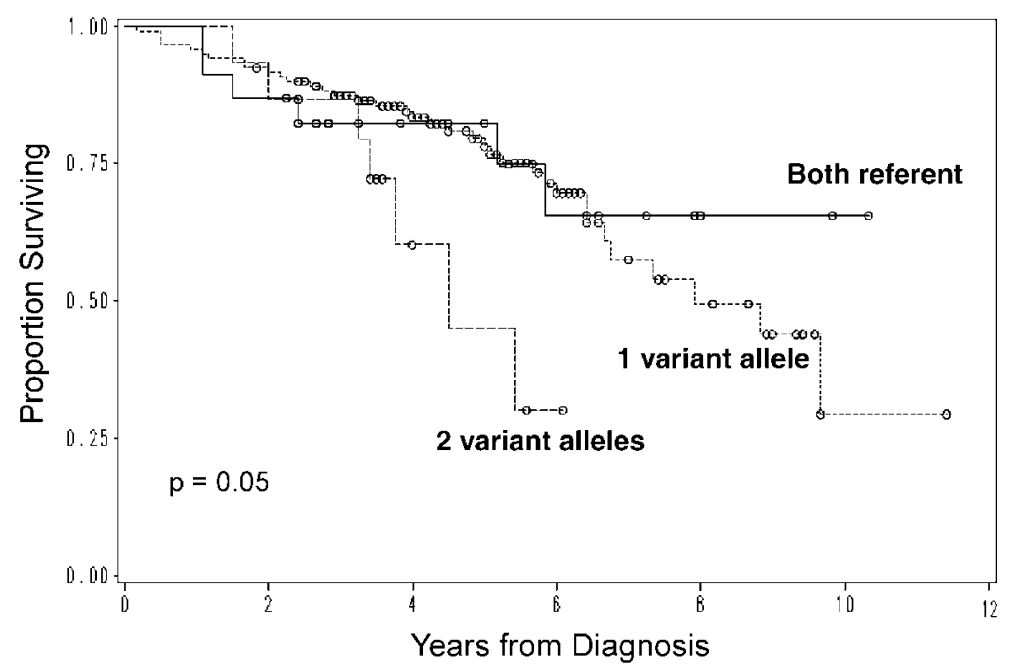

(b)

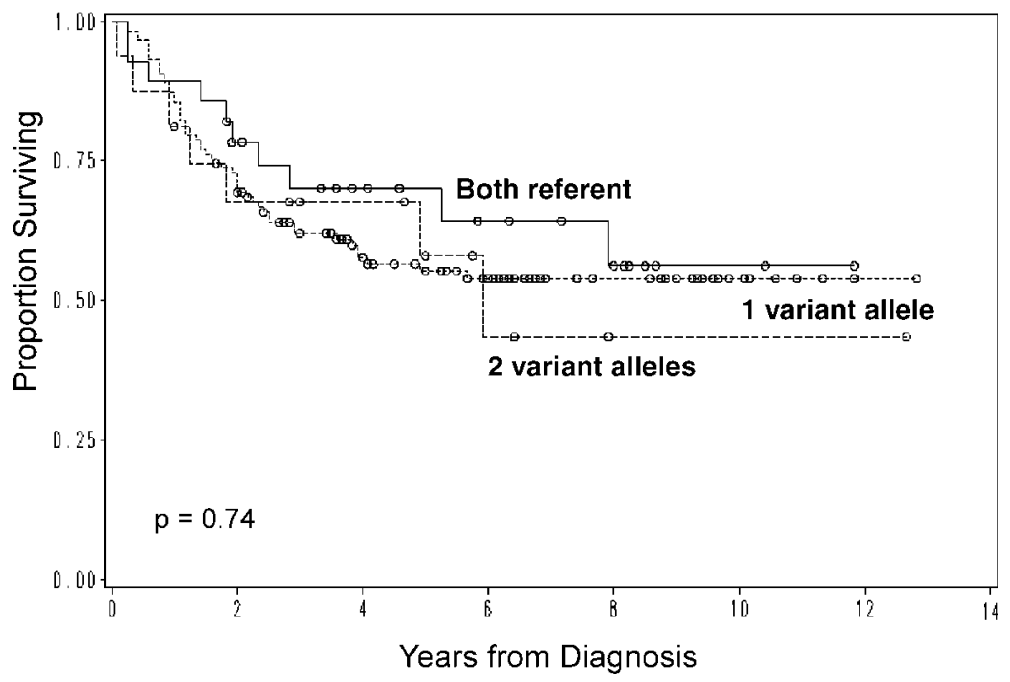

Figure 2. (a) Progression-free survival of breast cancer by combined UGT2B15 and SULT1A1 genotypes [35]. Patients receiving tamoxifen therapy; (b) patients not receiving tamoxifen as part of their treatment for breast cancer.

has been a mainstay of endocrine therapy for these patients for more than two decades. While the presence of ER and/or PR is a good marker for patients likely to benefit from antiestrogen therapy, not all patients who fall into the same category of receptor expression have the same degree of response to therapy. These differences in response are likely due, in part, to the individual biochemistry of the patient and may be explained by the contribution of genetic variation in metabolism (i.e. pharmacogenetics). The influence of genetic variation on response to drug therapy has long been recognized, but until recently, studies on the role of pharmacogenetics in relation to tamoxifen therapy have been lacking.

Several P450s are capable of producing 4-OH TAM while CYP2D6 has been shown to play the dominant role in the production of endoxifen, which is now considered to be the predominant active metabolite of tamoxifen since its pharmacological properties are equivalent to that of 4-OH TAM and its plasma con- centrations are eight-fold higher than that of $4-\mathrm{OH}$ TAM [10,22-24]. This metabolite is also produced by CYP2D6, and genetic variation in CYP2D6 has been shown to modulate plasma levels of endoxifen [24]. Therefore, genetic variation in CYP2D6 could be expected to influence efficacy of tamoxifen therapy. The CYP2D6*3 and CYP2D6*6 variants were rare in our study population (five individuals heterozygous for CYP2D6*3 and three individuals heterozygous for CYP2D6*6), therefore we were unable to analyze the contribution of these alleles in this study (data not shown). When CYP2D6*4 was examined, there was no detectable influence of this genotype on overall survival or recurrence of disease in either the patients who received tamoxifen therapy or those whose therapy did not include tamoxifen. It is interesting to note that in all subgroups, the CYP2D6*4 variant seemed to be associated with decreased risk of death or recurrence. Since none of the measures were statistically significant, the 
relevance of these findings is unclear. However, the study population was small and the polymorphism was found at a frequency of 0.15 in this population. Therefore, this study could be underpowered to detect the contribution of CYP2D6 to overall survival in breast cancer patients receiving tamoxifen, and these studies should be repeated in a larger study. Additionally, no information was available concerning concomitant medications, so it is possible that the findings are influenced by the patients receiving drugs that could inhibit the activity of CYP2D6. Another limitation is the fact that another CYP2D6 allele found commonly in African-Americans but not Caucasians could have an influence (25). Since only $20 \%$ of our study population was African-American, we were unable to examine the influence of this allele.

We also examined the contribution of genetic variation in UGT2B15 to overall survival and recurrence of disease. UDP-glucuronosyltransferases (UGTs) are Phase II detoxification enzymes that catalyze the transfer of the glucuronyl moiety from uridine $5^{\prime}$-diphosphoglucuronic acid to functional groups of a wide variety of endogenous and exogenous compounds, facilitating their excretion from the organism. The UGT2B family are products of separate genes whose isoforms preferentially glucuronidate steroids and bile acids, in addition to xenobiotics [26]. UGT2B15 catalyzes the biotransformation of a number of steroid substrates and exogenous compounds, including 4-OH TAM [27]. The UGT2B15*1/*2 polymorphism produces an amino acid change at residue 85 from aspartate $\left(\mathrm{D}^{85}\right)$ to tyrosine $\left(\mathrm{Y}^{85}\right)$, resulting in enzymes with similar $\mathrm{K}_{\mathrm{m}}$ values but with the $V_{\max }$ increased twofold in the case of the $\mathrm{Y}^{85}$ (UGT2B15*2) variant (15). UGT2B15 has been identified as the major UGT involved in the glucuronidation of the cis- isomer of 4-OH TAM. Early studies suggested that this isomer might exhibit estrogen agonist action $[28,29]$, but more recent studies have shown that, in the presence of estradiol, cis-4-OH TAM has a significant antagonist effect, although this effect is lower than that seen with the trans isomer $[9,30,31]$. It has also been reported that the geometrical isomerization of trans-4OH TAM to cis-4-OH TAM is catalyzed by CYP1B1 in an atypical P450 reaction [32]. However, when we examined a functional genetic polymorphism (Leu432Val) in CYP1B1 in this study population, there was no association with recurrence or survival in patients who did and did not receive tamoxifen (data not shown), indicating that isomerization does not substantially influence the pharmacological properties of 4-OH TAM or that the CYP1B1 polymorphism is not important enough in influencing CYP1B1 enzyme activity to show a difference. In this study, we found that the UGT2B15*2 high activity allele was associated with a non-significant $(p=0.11)$ increased recurrence of disease in the tamoxifen-treated patients, but not in those whose therapies did not include tamoxifen. This finding is as would be expected if an active metabolite was rapidly eliminated due to hepatic metabolism and excretion. UGT2B15 is expressed in the liver, where it is in a position to rapidly eliminate 4-OH TAM via the biliary system. The majority of tamoxifen and its metabolites are excreted in the bile [22,23]. Since sulfate conjugates of xenobiotics are mainly excreted in the urine, and glucuronides with molecular weights of greater than 350 are preferentially excreted in the bile, this indicates that glucuronidation may be the major excretory pathway. Therefore, increased glucuronidation of 4-OH TAM in the liver would lead to more rapid excretion of the active metabolite. Additionally, UGT2B15 is also expressed in adipose tissue in levels comparable to those found in other extrahepatic tissues [33], where it could participate in the extrahepatic metabolism of 4-OH TAM. Recently, it has been reported that another UGT, UGT1A4, is capable of directly glucuronidating tamoxifen [34]; hence, it is possible that genetic variation in UGT1A4 could play a role in response to tamoxifen. However, the $\mathrm{K}_{\mathrm{m}}$ for this reaction was relatively high $(32.4 \mu \mathrm{M})$, and the relative role of UGT1A4 in tamoxifen biotransformation remains to be established in vivo. The Phase II metabolism of endoxifen has not been specifically tested thus far, but it is very unlikely that the absence of one methyl group distant from the 4-hydroxy moiety would alter conjugation reactions catalyzed by UGT2B15 or SULT1A1. Additionally, genetic polymorphisms in these enzymes appear to influence efficacy of tamoxifen therapy, indicating their involvement in the metabolism of endoxifen.

Since SULT1A1 and UGT2B15 both catalyze Phase II conjugation reactions, and both have variant alleles that are associated with overall survival/recurrence in patients treated with tamoxifen, we also considered whether the combination of the two variants would have an effect on therapeutic outcomes. Individuals who possessed both variant alleles had significantly increased hazard of death compared to individuals with two common alleles, and these effects were greater than when the genes were examined separately. Likewise, there was a significant trend toward recurrence of disease with both variant alleles. When SULT1Al genotype was examined previously, it was the individuals who were homozygous for the low activity variant who had poorer overall survival. This finding was counterintuitive, if sulfation of 4-OH TAM is an excretory pathway. The epidemiological findings suggest that there is a unique mechanistic basis of the effect of sulfation of 4-OH TAM other than excretion. Studies are currently underway in our laboratory to elucidate the role of $\mathrm{SO}_{4}$-TAM in breast tumors (manuscript in preparation). However, if rapid sulfation is beneficial, and rapid glucuronidation is deleterious, then these variant alleles might be expected to have additive effects. In summary, we found that, while CYP2D6 genotype did not appear to influence therapeutic outcomes, polymorphisms in UGT2B15 and SULT1A1 do appear to have an effect on overall survival and recurrence of disease in patients treated with tamoxifen. However, this preliminary report needs to be replicated 
in a larger, more homogeneous study population. Because the study population consisted of women with various demographic and disease characteristics, who received a number of varied and repeated treatments in addition to tamoxifen, we are unable to determine if effects differ by disease stage, race, etc. Nonetheless, these findings indicate that genetic variability could provide an explanation for therapeutic failures.

\section{Acknowledgements}

The authors wish to acknowledge Bridgett Green, Angie Stone and Jason Plaxco for their technical assistance. This work was supported by Department of Defense DAMD17-02-1-0274, a grant from the Arkansas Breast Cancer Research Program, and in part by the National Institutes of General Medical Sciences grant GM61373 (UO1).

\section{References}

1. Furr BJ, Jordan VC: The pharmacology and clinical uses of tamoxifen. Pharmacol Ther 25: 127-205, 1984

2. Tamoxifen for early breast cancer: an overview of the randomised trials. Early Breast Cancer Trialists' Collaborative Group. Lancet, 351: 1451-1467, 1998

3. Kristensen B, Ejlertsen B, Dalgaard P, Larsen L, Holmegaard SN, Transbol I, Mouridsen HT: Tamoxifen and bone metabolism in postmenopausal low-risk breast cancer patients: a randomized study. J Clin Oncol, 12: 992-997, 1994

4. Love RR., Mazess RB, Barden HS, Epstein S, Newcomb PA, Jordan, VC, Carbone PP, DeMets DL: Effects of tamoxifen on bone mineral density in postmenopausal women with breast cancer. N Engl J Med, 326: 852-856, 1992

5. Ingram D: Tamoxifen use, oestrogen binding and serum lipids in postmenopausal women with breast cancer. Aust N Z J Surg, 60: 673-675, 1990

6. Bruning PF, Bonfrer JM, Hart AA, de Jong-Bakker M, Linders D, van Loon J, Nooyen WJ: Tamoxifen, serum lipoproteins and cardiovascular risk. Br J Cancer, 58: 497-499, 1988

7. Braithwaite RS, Chlebowski RT, Lau J, George S, Hess R, Col NF: Meta-analysis of vascular and neoplastic events associated with tamoxifen. J Gen Intern Med, 18: 937-947, 2003

8. Desta Z, Ward BA, Soukhova NV, Flockhart DA: Comprehensive evaluation of tamoxifen sequential biotransformation by the human cytochrome $\mathrm{P} 450$ system in vitro: prominent roles for CYP3A and CYP2D6. J Pharmacol Exp Ther, 2004

9. Jordan VC, Koch R, Langan S, McCague R: Ligand interaction at the estrogen receptor to program antiestrogen action: a study with nonsteroidal compounds in vitro. Endocrinology, 122: 1449-1454, 1988

10. Johnson MD, Zuo H, Lee KH, Trebley JP, Rae JM, Weatherman RV, Desta Z, Flockhart DA, Skaar TC: Pharmacological characterization of 4-hydroxy- $N$-desmethyl tamoxifen, a novel active metabolite of tamoxifen. Breast Cancer Res Treat 85: 151-159, 2004

11. Sachse C, Brockmoller J, Bauer S, Roots I: Cytochrome P450 2D6 variants in a Caucasian population: allele frequencies and phenotypic consequences. Am J Hum Genet 60: 284-295, 1997

12. Nishiyama $T$, Ogura $K$, Nakano $H$, Ohnuma $T$, Kaku $T$, Hiratsuka A, Muro K, Watabe T: Reverse geometrical selectivity in glucuronidation and sulfation of cis- and trans-4-hydroxytamoxifens by human liver UDP-glucuronosyltransferases and sulfotransferases. Biochem Pharmacol 63: 1817-1830, 2002
13. Raftogianis RB, Wood TC, Otterness DM, Van Loon JA, Weinshilboum, RM: Phenol sulfotransferase pharmacogenetics in humans: association of common SULT1A1 alleles with TS PST phenotype. Biochem Biophys Res Commun 239: 298-304, 1997

14. Nowell S, Ambrosone CB, Ozawa S, MacLeod SL, Mrackova G, Williams S, Plaxco J, Kadlubar FF, Lang NP: Relationship of phenol sulfotransferase activity (SULT1A1) genotype to sulfotransferase phenotype in platelet cytosol. Pharmacogenetics 10 : 789-797, 2000

15. Levesque E, Beaulieu M, Green MD, Tephly TR, Belanger A, Hum DW: Isolation and characterization of UGT2B15(Y85): a UDPglucuronosyltransferase encoded by a polymorphic gene. Pharmacogenetics 7: 317-325, 1997

16. Nowell S, Sweeney C, Winters M, Stone A, Lang NP, Hutchins LF, Kadlubar FF, Ambrosone CB: Association between sulfotransferase $1 \mathrm{~A} 1$ genotype and survival of breast cancer patients receiving tamoxifen therapy. J Natl Cancer Inst 94: 1635-1640, 2002

17. Ambrosone CB, Sweeney C, Coles BF, Thompson PA, McClure GY, Korourian S, Fares MY, Stone A, Kadlubar FF, Hutchins LF: Polymorphisms in glutathione S-transferases (GSTM1 and GSTT1) and survival after treatment for breast cancer. Cancer Res 61: 7130-7135, 2001

18. Liu Q, Thorland EC, Heit JA, Sommer SS: Overlapping PCR for bidirectional PCR amplification of specific alleles: a rapid one-tube method for simultaneously differentiating homozygotes and heterozygotes. Genome Res 7: 389-398, 1997

19. Ries LAG, Eisner MP, Kosary CL, Hankey BF, Miller BA, Clegg L, Mariotto A, Fay MP, Feuer EJ, Edwards BK (eds). SEER Cancer Statistics Review, 1975-2000. National Cancer Institute, Bethesda, MD, 2003

20. Chu KC, Tarone RE, Kessler LG, Ries LA, Hankey BF, Miller BA, Edwards BK: Recent trends in U.S. breast cancer incidence, survival, and mortality rates. J Natl Cancer Inst 88: 1571-1579, 1996

21. American Cancer Society. Cancer Facts and Figures. 2003

22. Lien EA, Solheim E, Kvinnsland S, Ueland PM: Identification of 4-hydroxy- $N$-desmethyltamoxifen as a metabolite of tamoxifen in human bile. Cancer Res 48: 2304-2308, 1988

23. Lien EA, Solheim E, Lea OA, Lundgren S, Kvinnsland S, Ueland PM: Distribution of 4-hydroxy-N-desmethyltamoxifen and other tamoxifen metabolites in human biological fluids during tamoxifen treatment. Cancer Res 49: 2175-2183, 1989

24. Stearns V, Johnson MD, Rae JM, Morocho A, Novielli A, Bhargava P, Hayes DF, Desta Z, Flockhart DA: Active tamoxifen metabolite plasma concentrations after coadministration of tamoxifen and the selective serotonin reuptake inhibitor paroxetine. J Natl Cancer Inst 95: 1758-1764, 2003

25. Bradford LD, Gaedigk A, Leeder JS: High frequency of CYP2D6 poor and "intermediate" metabolizers in black populations: a review and preliminary data. Psychopharmacol Bull 34: 797-804, 1998

26. Burchell B, Nebert DW, Nelson DR, Bock KW, Iyanagi T, Jansen PL, Lancet D, Mulder GJ, Chowdhury JR, Siest G et al. The UDP glucuronosyltransferase gene superfamily: suggested nomenclature based on evolutionary divergence. DNA Cell Biol 10: 487-494, 1991

27. Green MD, Oturu EM, Tephly TR: Stable expression of a human liver UDP-glucuronosyltransferase (UGT2B15) with activity toward steroid and xenobiotic substrates. Drug Metab Dispos 22: 799-805, 1994

28. Katzenellenbogen JA, Carlson KE, Katzenellenbogen BS: Facile geometric isomerization of phenolic non-steroidal estrogens and antiestrogens: limitations to the interpretation of experiments characterizing the activity of individual isomers. J Steroid Biochem 22: 589-596, 1985

29. Jordan VC, Haldemann B, Allen KE: Geometric isomers of substituted triphenylethylenes and antiestrogen action. Endocrinology 108: 1353-1361, 1981

30. Murphy CS, Langan-Fahey SM, McCague R, Jordan VC: Structure-function relationships of hydroxylated metabolites of 
tamoxifen that control the proliferation of estrogen-responsive T47D breast cancer cells in vitro. Mol Pharmacol 38: 737-743, 1990

31. Malet C, Spritzer P, Cumins C, Guillaumin D, Mauvais-Jarvis $P$, Kuttenn F: Effect of 4-hydroxytamoxifen isomers on growth and ultrastructural aspects of normal human breast epithelial (HBE) cells in culture. J Steroid Biochem Mol Biol 82: 289-296, 2002

32. Crewe HK, Notley LM, Wunsch RM, Lennard MS, Gillam EM: Metabolism of tamoxifen by recombinant human cytochrome P450 enzymes: formation of the 4-hydroxy, 4'-hydroxy and $\mathrm{N}$-desmethyl metabolites and isomerization of trans-4-hydroxytamoxifen. Drug Metab Dispos 30: 869-874, 2002

33. Tchernof A, Levesque E, Beaulieu M, Couture P, Despres JP, Hum DW, Belanger A: Expression of the androgen metabolizing enzyme UGT2B15 in adipose tissue and relative expression measurement using a competitive RT-PCR method. Clin Endocrinol (Oxf) 50: 637-642, 1999

34. Kaku T, Ogura K, Nishiyama T, Ohnuma T, Muro K, Hiratsuka A: Quaternary ammonium-linked glucuronidation of tamoxifen by human liver microsomes and UDP-glucuronosyltransferase 1A4. Biochem Pharmacol 67: 2093-2102, 2004

35. Ambrosone CB, JA, HF, CS, AT: Variants in Oxidative StressRelated Genes (MnSOD, MPO) and Survival after Treatment for Breast Cancer. Proc Am Assoc Cancer Res 2004

Address for offprints and correspondence: Susan Nowell, $\mathrm{PhD}$, Roswell Park Cancer Institute, BSB Room S701, Elm \& Carlton Streets, Buffalo, NY 14263, USA; Tel.: +1-716-845-3103; Fax:+1-716-845-1356; E-mail: Susan.Nowell@RoswellPark.org 